\title{
LOGISTICS CHAIN ANALYSIS BASED ON A BELIEF NETWORK
}

\author{
Clara Savchenko \\ Specialized Institute of Intelligent Computer Technologies \\ Technical University of Riga \\ I, Kalkyu Street, Riga LV - 1658, Latvia \\ Phone: +3717320019 \\ Fax: +3717820094
}

\section{STATEMENT OF THE PROBLEM}

In the present paper, the concept of logistics is used to construct a decision making system for choosing the best logistics chain. In principle, the logistic approach to the management of material flows differs from the conventional one in following: it integrates separate elements into the common system that is able to react adequately to the perturbation of the environment (Coyle, Bardi et al. 1992).

Logistics operations on the way of material flow movement from the initial source of raw materials to the terminal consumer are mostly fulfilled by using various transport vehicles. Costs for performing these operations constitute $50 \%$ of the total amount of logistics costs.

Logistics chain is a linearly ordered set of participants of the logistic process that implement logistics operations on transportation of the external material flow from one logistics system to another (see Figure.1).

The presence of an uncertain decision making situation, probabilistic data changing as the time changes, complex relationships among the variables and the subjectivity of task perception in the given problem make it necessary to use an influence diagram (Moravski 1989) as a model of decision making problem. 


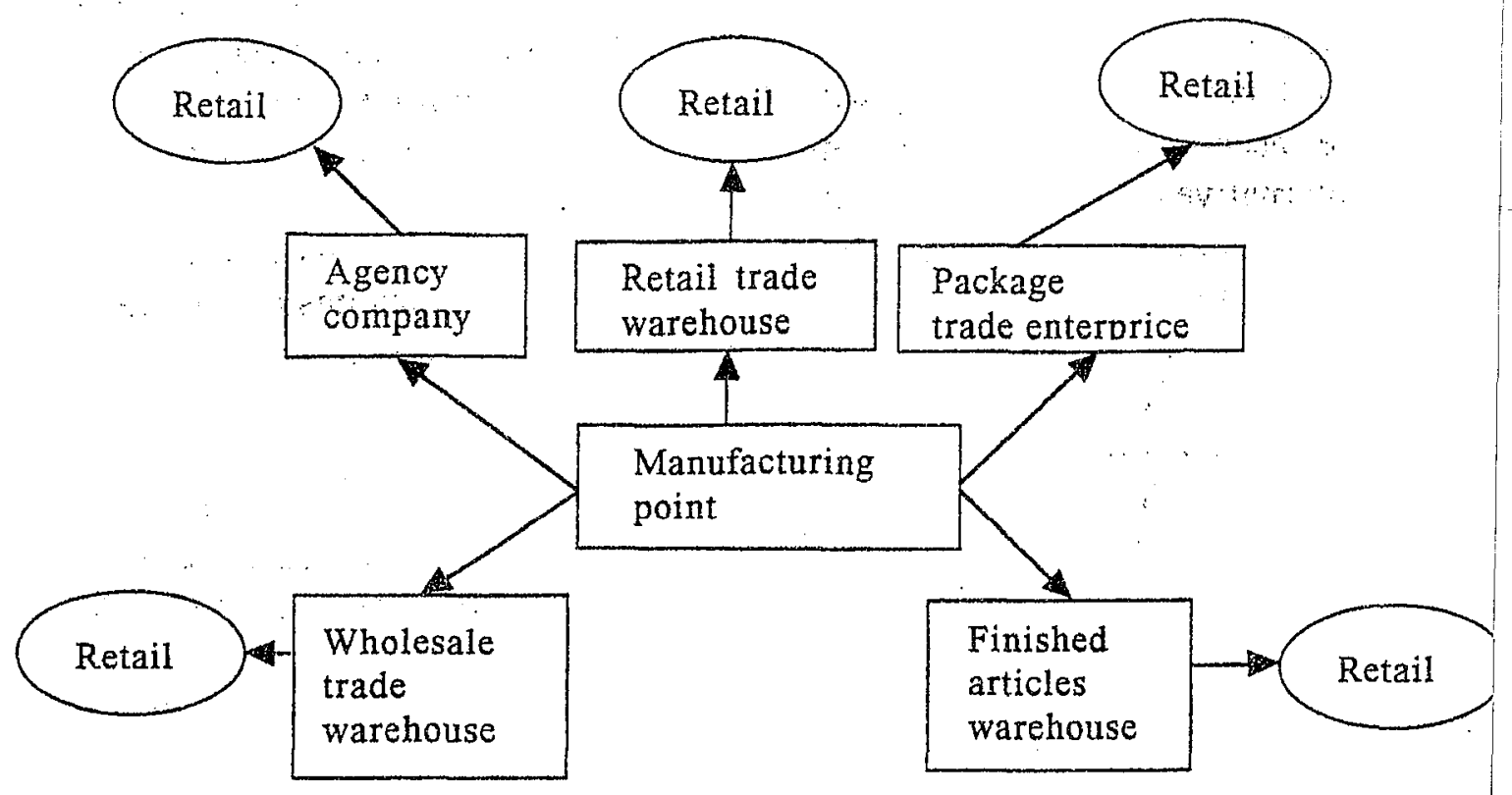

Fig. 1. Various consumer goods distribution channels.

\section{INFLUENCE DIAGRAM GENERATION}

To construct an influence diagram, let us use the goal-oriented influence diagram generation. First, it is necessary to determine possible values of a decision note. The complexity of a logistics chain depends essentially on goods transportation modes. Given own access roads, necessary transports and loading / unloading means, a logistics chain consists of two participants only, i.e. the seller and the customer.

In the problem under consideration, in order to deliver goods from the manufacturer to the customer, it is necessary to apply to a forwarding firm, since the customer, has no his own transport. With this, it should be taken into account that transportation by two transports complicates the logistics chain significantly as automobile transport is often involved in such transportations and sometimes an intermediary, the warehouse owner, is drawn in. Goods transportation is simplified essentially when direct relationship between the manufacturer-seller and the customers is broken by a transportation intermediary. Such a chain is average in complexity, easy arrangable and easy implementable.

To solve the problem under consideration, let us apply a logistics chain consisting of three participants: a manufacturer-seller, a forwarding 
intermediary and a wholesale base-customer. A manufacturer-seller and a wholesale base-customer are constant elements of the chain, whereas several forwarding firms can stand for a forwarding intermediary. Thus, the forwarder-intermediary will figure as a decision node. Then it should be ascertained for which aim the decision is made and how the alternatives available will be evaluated.

Since the problem is to choose a logistics chain, let us ascertain for which aim the chain is created. Any activity in logistics has a finite goal that was called six logistics rules. They are: (1) cargo is reqired cargo, (2) quality is the required quality, (3) quantity is necessary amount, (4) time is required time, (5) destination is the required destination, (6) costs are minimal. The aim of a logistics chain is considered to be achieved if all the six conditions are met. Let us call the degree of fulfilling those six conditions the efficiency of a logistics chain. Assume that the manufacture-seller and the customer do not influence the increase of the logistics chain efficiency as they are constant elements of a logistics chain. The efficiency of a logistics chain will depend on the forwarding firm's activity. That is, the estimation of a forwarding firm activity's efficiency will be used as the evaluation of available alternatives and will be regarded as a value node.

Since a decision node and a value node are determined, the minimal influence diagram could be constructed (see Fig. 2).

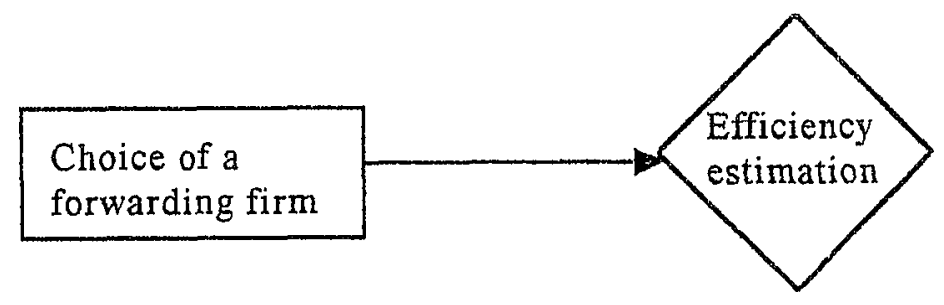

Fig. 2. An influence diagram for choosing a forwarding firm.

At the next stage, it should be determined which chance nodes are necessary for the estimation of the value node.

Since the efficiency of a logistics chain depends on the above six logistics rules fulfillment, the influence diagram should then be supplemented with following chance nodes: goods, quality, quantity, full delivery time, destination and costs. Let us determine values of generated 
chance nodes. Chance node "Required goods" can assume two values: "yes" or "no" that depend on the possibility to transport necessary goods by a forwarding firm's transport.

Chance node "Destination" can also assume two values: "yes" or "no" which depend on the possibility to deliver goods by a forwarding firm to the required place. A chance node "Costs" can assurne several values measured in monetary units depending on the distance, quantity of transported goods and transportation rate.

Values of a chance node "Full delivery time" measured in hours depend on transportation time and transport delays. Values of a chance node "Quantity", measured in per cents of the shipped cargo, depend on the goods safety on the way. Values of a chance node "Quality" depend on the quality of spoiled goods and are also measured in per cents. An influence diagram should now be supplemented with new chance nodes. Chance nodes "Possibility to transport" and "Possibility to deliver" can assume two values: "yes" and "no" and are determined depending on the chosen transport.

A chance node "Delay on the way" is measured in days and has some prior probability for cargo delay on the way, however this probability could be ascertained given information on the weather for the planned period. Transport delay can be also caused by an accident. A chance node "Delay on the way" is decomposed into two nodes: "Accident" and "Weather". The chance of an accident, in its turn, depends on the technical state of transport. It can be unsatisfactory, satisfactory, normal and excellent.

As goods shipment frequency is an important factor when choosing a transport vehicle, the influence diagram is supplemented with a chance node "Shipment frequency" that is measured in shipment times per week. The choice of shipment type is influenced by convenient goods delivery from manufacturing point to the starting point and from destination point to the wholesale base. Then one more chance node, "Convenient delivery", is incorporated, the qualitative characteristic for which could be evaluated in points. It should also be taken into account that delivery time depends on the transportation distance. Taking into account that delivery time depends on the distance but spoiling of goods depends on the accident, nodes "Delivery time" and "Transportation distance" should be connected by directed in pairs arcs. All boundary nodes of such influence diagram are of deterministic or probabilistic nature. Thus there is no need to incorporate any new nodes, since the influence diagram is 
complete and finished. Generation of an influence diagram for the choice of a forwarding firm is considered to be completed.

\section{DETERMINATION OF POSSIBLE DECISION OPTIONS}

Two decisions should be made in the problem under consideration. They are: to choose a forwarding firm and to choose goods transportation type. Since both decisions are interconnected, combinations of forwarding firm choice options and transportation types could be regarded as decision options.

Let us find an allowable set of alternative combinations of decisions about the choice of a forwarding firm and transportation form taking into account that the goods to be shipped are washing machines, i.e. a big cargo that can be shipped both in containers and in small packages according to the total goods weight. Then, an assumption will be made for the present problem that between the starting point and the destination point there exist all kinds of transport communications. Transportation can be made by several firms dealing with cargo transportations. Among them there could be: a) a firm transporting goods by motor transport (forwarder 1), b) a firm transporting goods by rail (forwarder 2), c) a firm transporting goods by air (forwarder 3), and d) a firm transporting goods by over water (forwarder 4). A transportation contract will be concluded with a single firm. An option of transport vehicles combination is not considered in order to avoid problems of coordinated operation of two intermediary firms and due to additional loading / unloading costs.

\section{INFLUENCE DIAGRAM REDUCTION}

Let us analyse the obtained influence diagram for the choice of a forwarding firm. The possibility of delivering required goods to the required destination point is the necessary condition during construcing a logistics chain. In case if eithe of Variables "Transportation possibility" or "Delivery possibility" is equal to zero, the efficiency of the logistics chain will also equal zero. Values of those variables are known to the decision maker before a decision is made. All this influences the choice of an alternative decision option. Thus the dependence of a value node on 
variables "Transportation possibility" and "Delivery possibility" can be taken off. With this, limitations on the choice of alternatives should placed. There should exist a possibility of delivering goods from the starting point to the destination point by using transport that a forwarding firm, which is a possible alternative decision, possesses. Besides, this kind of transport is to be adjusted for transporting the goods mentioned in the contract.

In the obtained influence diagram, variables "Convenient delivery", "Theft of cargo" and "Shipment frequency" have constant values for each decision option. Calculations for variables "Costs" and "Full delivery time" are given above. It is necessary to determine probability distributions for nodes "Spoiling of cargo" and "Delay on the way" only. To find them, a belief network (see Figure 3 ) is used that is transformed from an influence diagram by extracting value and decision nodes. Deterministic chance nodes "Convenient delivery", "Theft of cargo", "Shipment frequency" and "Costs" were not included into the belief network.

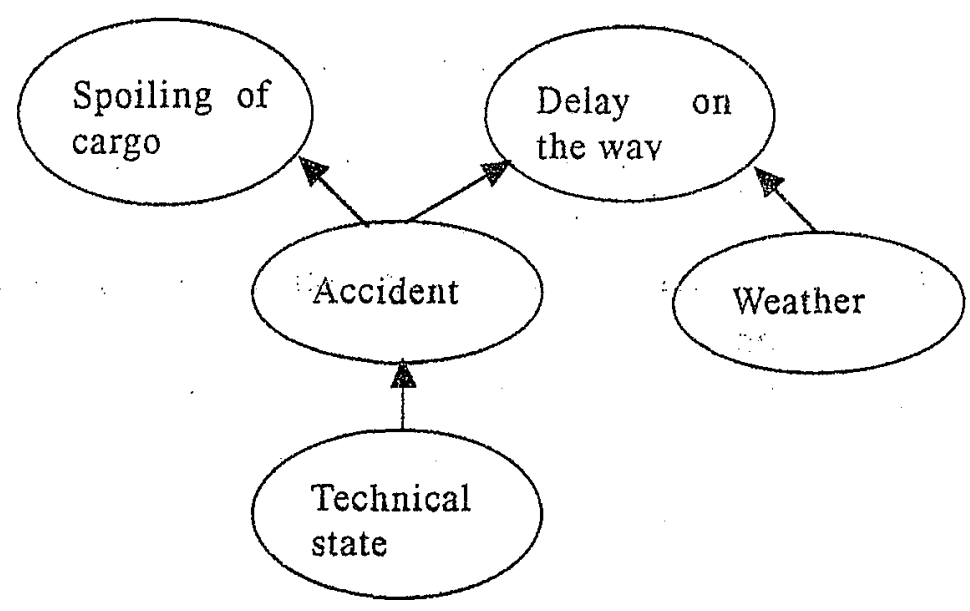

Fig.3. A belief network for the choice of a forwarding firm.

\section{REFERENCES}

The management of business logistics. / J. J. Coyle, E. J. Bardi and C. J. Langley.- St. Paul: West Publishing Company, 1992.

Morawski P. Understanding Bayesian belief networks. AI Expert. - May 1989. - 44. - 48. p. 